\title{
СТРУКТУРНЫЕ ПАРАМЕТРЫ ПРОДУКТОВ ТЕРМОДЕСТРУКЦИИ УГЛЕВОДНЫХ СИСТЕМ И ИХ ВЗАИМОСВЯЗЬ С БИОАКТИВНОСТЬЮ
}

\author{
И.С. Черепанов, кандидат химических наук, доцент
}

Удмуртский государственный университет

E-mail: cherchem@mail.ru

Ключевые слова: D-мальтоза, карбонильные соединения, гетероциклы, ИК Фурье-спектроскопия, карамелизация, масс-спектрометрия, структура, анализ.

Реферат. Реакции деструкции и распада углеводов, наблюдающиеся в пищевых технологиях при высокотемпературной обработке сырья, приводят к продуктам, способным проявлять биологическую активность различного характера. Последнее зависит от функиионально-группового состава продуктов термической деструкиии, что предполагает актуальность изучения закономерностей формирования их структуры в различных режимах процессов обработки углеводов. В настоящей работе представлены результаты исследований по изучению образования продуктов термодеструкиии $D$-мальтозы и полуколичественной оченке содержания структурных элементов, влияюших на проявление биоактивности. Методами ИК Фурье-спектроскопии и пиролитической масс-спектрометрии показано, что в процессах щелочного распада и сухой карамелизации исходного углевода формируются окрашенные структуры, содержащие предельные и непредельные алифатические фрагменты, конденсированные с гетероциклами различной степени замещенности. Установлено также, что сухая термодеструкиия, требующая жесткого температурного режима, приводит к более глубоким структурным изменениям в процессе воздействия в сравнении с реакциями карамелизации в растворах. В рамках двухпараметрической модели показана возможность количественной оценки структурных составляющих продуктов деструкции мальтозы в различных условиях, показано преобладание непредельной и гетерочиклической составляющей структуры продуктов сухой термодеструкиии исходного углевода. Результаты исследований имеют практическое значение при исследовании структуры и прогнозировании свойств и характера биоактивности продуктов переработки углеводного сырья в пищевой промышленности.

Реакции деструкции и распада углеводов имеют важное практическое значение не только в пищевых технологиях при высокотемпературной обработке сырья [1], но и являются в настоящее время эффективной синтетической платформой получения различных низкомолекулярных веществ [2] и полимерных продуктов целевого назначения [3]. Другой востребованной областью применения химически и термически трансформированных углеводов является получение экологически чистых и дешевых природных красителей, поскольку продукты хемо- и термодеструкции сахаров интенсивно окрашены и обладают хорошей адгезией ко многим волокнистым материалам [3]. При этом продукты термодеструкции могут оказывать физиологическое действие различного характера [4]. Непредельная составляющая структуры обусловливает антиокислительную активность и склонность к старению, фурановые циклы при энзиматическом окислении

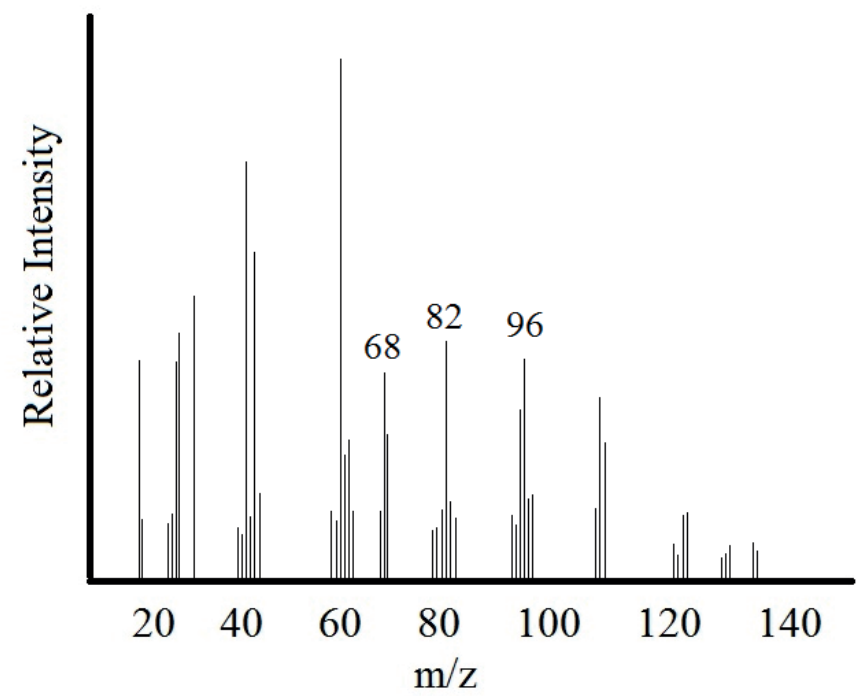

Puc. 1. PY-MS-спектр конденсированной фазы продуктов щелочного распада мальтозы 
в организмах образуют активные метаболиты, способные алкилировать белки и нуклеиновые кислоты, проявляя токсическое действие [5]. Перечисленные свойства, очевидно, зависят от функционально-группового состава продуктов термической деструкции, что предполагает актуальность изучения закономерностей формирования структуры продуктов в различных режимах процессов обработки углеводов.

Изучение строения продуктов трансформации углеводов представляет собой нетривиальную задачу, поскольку распад и последующие процессы усложнения структуры протекают по нескольким параллельным направлениям и могут варьироваться в зависимости от условий

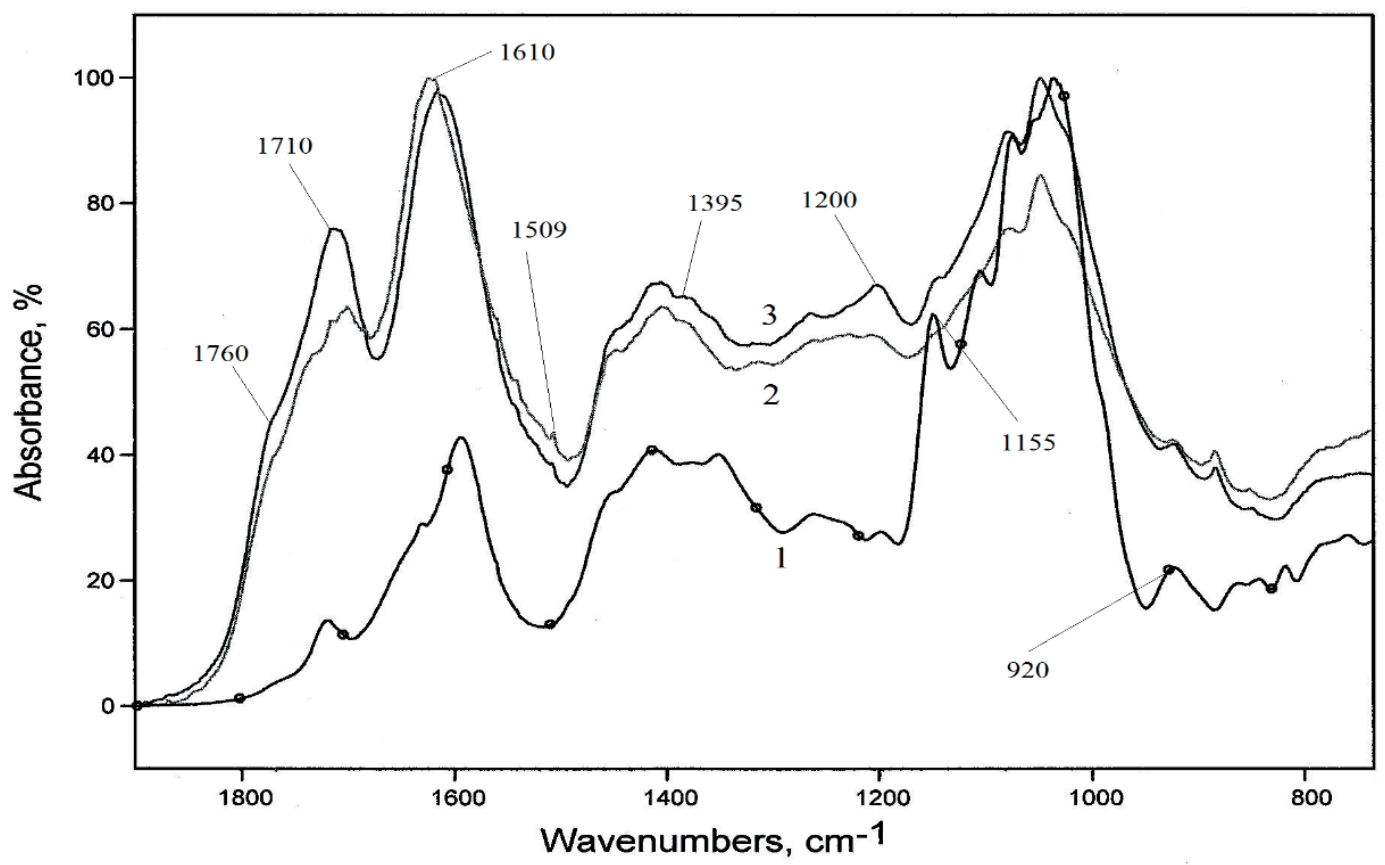

Puc. 2. Фрагменты ИК Фурье-спектров конденсированных продуктов термодеструкции мальтозы (KBr, $1: 200)$ : 1 - щелочной распад; 2 - сухая термодеструкция продуктов щелочного распада; 3 - сухая термодеструкция исходного углевода

[1-3]. Традиционно интерес исследователей направлен на идентификацию и изучение широкого спектра низкомолекулярных продуктов карамелизации (caramel aroma) методами хромато-масс-спектрометрии и ЯМР $[2,6]$, тогда как конденсированные продукты распада углеводов (caramel color) в различных условиях изучены в меньшей степени $[3,7,8]$. При этом анализ литературных данных показывает, что объектами исследований чаще всего являются только наиболее распространенные углеводы, такие как глюкоза [2], лактоза [1] и сахароза [3], в то время как структурные отличия разных сахаров обусловливают разницу в химическом поведении [9]. Одним из важнейших, но малоизученных углеводов является D-мальтоза, имеющая высокую энергетическую ценность, применяющаяся в качестве компонентов детского и спортивного питания, низкоаллергенного заменителя сахарозы. Ранее нами были изучены начальные стадии формирования структуры продуктов щелочной карамелизации D-мальтозы [7], в связи с чем целью настоящей работы являлось:

- изучение образования и природы продуктов карамелизации мальтозы в жестких условиях сухой термодеструкции, моделирующей высокотемпературную технологическую обработку пищевых продуктов и растительного сырья;

- установление для выделенных карамельных составов полуколичественных корреляций «структура - токсичность» на основе анализа ИК Фурье-спектров. 
Конденсированные продукты щелочной деструкции были получены термостатированием $\left(85 \pm 2{ }^{\circ} \mathrm{C}\right)$ водно-этанольных (60 \% EtOH, Merck) растворов D-мальтозы (Sigma-Aldrich, 0,002 моль в 20 мл растворителя) в присутствии $3 \cdot 10^{-4}$ моль щелочи. В процессе синтеза снимали электронные спектры (спектрофотометр СФ-2000) разбавленных проб для контроля за ходом реакции. Через 1,5 ч термостатирования растворитель удаляли на роторном испарителе, твердую фазу высушивали, дважды промывали эфиром и подвергали сухой термодеструкции $\left(150{ }^{\circ} \mathrm{C}\right)$ в течение 1 ч при атмосферном давлении. В аналогичных условиях проводилась сухая карамелизация исходного углевода. Колебательные спектры всех твердых продуктов снимали на ИК Фурье-спектрометре ФСМ-2201 в таблетках бромида калия $(1: 200)$ в интервале волновых чисел 4000-400 см с $^{-1}$ со спектральным разрешением $4 \mathrm{~cm}^{-1}$ при 40 сканах и обрабатывали в программе FSpec. Интенсивность полос поглощения определяли по методу базовой линии. Кратность измерений составляла не менее трех экспериментов. Масс-спектрометрические измерения проводили на масс-спектрометре Varian MAT 311A с пиролитической ячейкой $\left(250{ }^{\circ} \mathrm{C}\right)$.

Ранее было показано [7], что структурные фрагменты структуры конденсированных продуктов в мягких условиях щелочного распада в этанольной среде представляют собой сочетание нетрансформированных пиранозных циклов и функционализированных хромофорных фрагментов $\left(\lambda_{\max }=280,320,410 \mathrm{HM}\right)$.

Macc-спектр (PY-MS, $250{ }^{\circ} \mathrm{C}$ ) конденсированной фазы, полученной в результате щелочного распада мальтозы в водно-этанольном растворе, представлен на рис. 1.

Основные сигналы в масс-спектре характеризуют низкомолекулярные продукты термораспада, в частности пики $\mathrm{m} / \mathrm{z}=68,82,96$ характеризуют фурановые соединения [6], пикам с меньшими $\mathrm{m} / \mathrm{z}$ отвечают $\mathrm{C}_{1}, \mathrm{C}_{2}$ низкомолекулярные летучие продукты.

В целом основные сигналы аналогичны таковым для масс-спектров продуктов термодеструкции дисахаридов, подтверждающих образование гетероциклических производных в процессах «сухой» карамелизации $[1,6]$. С целью детализации закономерностей процессов формирования структуры на основании данных колебательной спектроскопии было проанализировано строение полученных в различных условиях продуктов термораспада. Анализ профилей ИК Фурье-спектров продуктов щелочного распада (рис. 2, спектр 1) показывает наличие существенной составляющей нетрансформированного углевода, о чем свидетельствуют полосы $1360,1270\left(\delta_{\mathrm{CCH}}, \delta_{\text {Осн }}\right), 1155$ (гликозидные связи), 1100 ( $v_{\mathrm{C}-\mathrm{O}}$ вторичных С-ОН) и 900$940 \mathrm{~cm}^{-1}$ (пиранозные кольца) [2, 3, 7]. Об этом же говорит низкое отношение интегральных интенсивностей полос поглощения $\mathrm{I}_{1720-1600} / \mathrm{I}_{1200-1000}$.<smiles>[R]C(=O)CC(=O)/C=C(/O)[In]</smiles>

caramel structure fragment

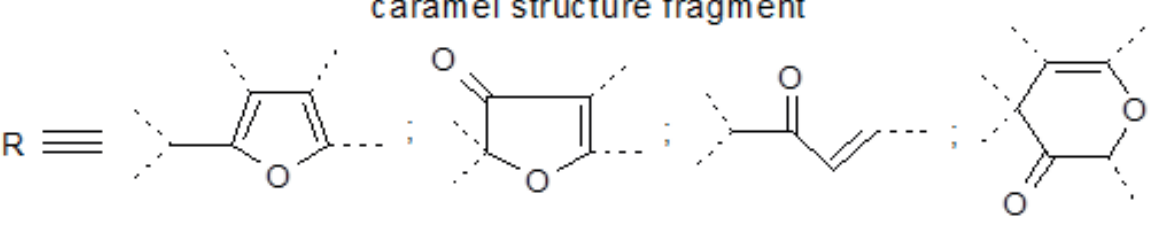

Несколько иная картина наблюдается при анализе спектров продуктов сухой термодеструкции (см. рис. 2, спектры 2, 3). Существенно возрастает интенсивность карбонильной полосы 1700-1770 см-1 ( $\left.v_{\mathrm{C}=\mathrm{O}}\right)$, при этом снижается интенсивность полос 1155,1100 и 920 см$^{-1}$, что свидетельствует о расщеплении гликозидных связей и дальнейшей трансформации пиранозных фрагментов. Комбинация полос в интервале 1600-1610 и $1710 \mathrm{~cm}^{-1}$ относится к колебаниям фрагментов, сопряженных с фурановыми циклами $\mathrm{C}=\mathrm{C}-\mathrm{CO}-\mathrm{C}=\mathrm{C}$-систем [10]. Кроме того, по- 
является плечо при $1760 \mathrm{~cm}^{-1}$, что может отвечать образованию сложноэфирных групп, полосы 1395 и 1200 см$^{-1}$ отвечают валентным колебаниям C-O-C и C-O+C-C фрагментов фурановых эфиров $[2,3]$.

$$
A-\text { factor }=\frac{I_{2920}+I_{2855}}{I_{2920}+I_{2855}+I_{1610}} \quad(1) \quad C-\text { factor }=\frac{I_{1710}}{I_{1710}+I_{1610}}
$$

Усиление полос в области $1430 \mathrm{~cm}^{-1}\left(\delta_{\mathrm{C-H}}\right)$ указывает на наличие соседних с метиленовыми группами кратных связей [11]. Слабая полоса при 1509 см $^{-1}$ относится к валентным колебаниям $\mathrm{C}=\mathrm{C}$-связей $\alpha$-замещенного фуранового кольца $[2,3,12]$ и более четко выражена в спектре продуктов сухой термодеструкции продуктов щелочного распада. Кроме того, для этой же системы отмечается наличие полос при $1700 \mathrm{~cm}^{-1}$ (оксогруппа в $\beta$-положении гетероцикла [13]), а также в области 1710-1740 $\mathrm{cm}^{-1}$, относимых, вероятно, к колебаниям $\mathrm{C}=\mathrm{O}$ оксопроизводных пиранов $[14,15]$, образование которых происходит из пиранозных циклов как результат некаталитических процессов [6]. Низкая интенсивность полос $885 \mathrm{~cm}^{-1}\left(\delta_{=\mathrm{C}-\mathrm{H}}\right)$ указывает на
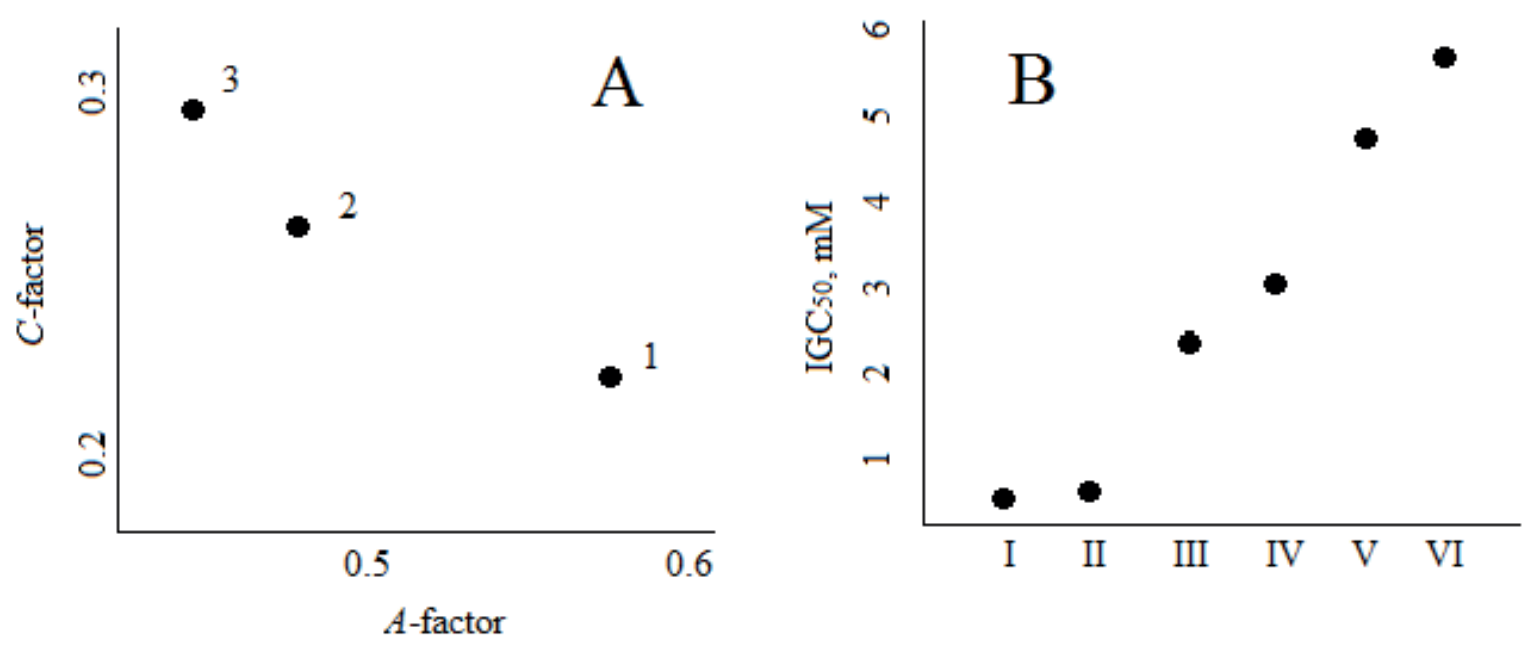

Puc. 3. Количественная оценка структурных параметров продуктов термодеструкции углеводных систем: 1 - щелочной распад; 2 - сухая термодеструкция продуктов щелочного распада; 3 - сухая термодеструкция исходного углевода (А).

Корреляция «структура - токсичность $\alpha, \beta$-непредельных карбонильных соединений» $[20]: \mathrm{I}-\mathrm{O}=\mathrm{CC}=\mathrm{CCCCCC}$; II $-\mathrm{CC}(=\mathrm{O}) \mathrm{C}=\mathrm{CCCCC}$; III $-\mathrm{CCC}(=\mathrm{O}) \mathrm{CC}=\mathrm{CCC} ; \mathrm{IV}-\mathrm{C}=\mathrm{CC}(=\mathrm{O}) \mathrm{OCCCC} ; \mathrm{V}-\mathrm{CC}(=\mathrm{O}) \mathrm{C}=\mathrm{CCC}$;

$$
\mathrm{VI}-\mathrm{CCOC}(=\mathrm{O}) \mathrm{C}=\mathrm{CC}(\mathrm{B})
$$

$\alpha, \alpha^{\prime}$-замещение фуранового цикла $[13,16]$, при этом усиление поглощения при 790-800 $\mathrm{cm}^{-1}$ свидетельствует об увеличении замещения фуранового кольца [12, 17], наиболее четко проявляющемся в спектре 2.

Таким образом, сухая термодеструкция, требующая жесткого температурного режима, приводит к более глубоким структурным изменениям в процессе воздействия. Близость профилей полос спектров продуктов термодеструкции исходного и карамелизованного углеводов (см. рис. 2, спектры 2, 3) свидетельствует о схожести механизмов превращения углевода в «сухих» условиях и в растворе, при этом соотношения структурных элементов, вероятно, остаются различными [8]. Проведенный анализ данных показывает, что конденсированные продукты карамелизации углеводов вряд ли представляют собой регулярные структуры, как на это указывается L. Kroh [8], более вероятно их строение в виде конденсированной системы гетероциклических колец различной степени замещенности.

На основании представленного выше анализа данных колебательной спектроскопии можно предположить следующую схему протекания целевых процессов. Сухая термодеструкция 
дисахаридов начинается, как известно, с расщепления гликозидной связи [8], которое в молекуле мальтозы в условиях термодеструкции протекает легче, чем у многих других ди- и олигосахаридов [9] с внутримолекулярной активацией [18].

Образующиеся на начальных этапах глюкоза и ангидроглюкоза претерпевают дальнейшие превращения, протекающие по различным направлениям (изомеризация, дегидратация, распад), при этом 1,6-ангидроглюкоза, по данным L. Kroh [8], дает в основном низкомолекулярные летучие производные фурана, в частности фурфурол, а также 5-гидроксиметилфурфурол, фуриловый спирт и другие $\alpha$ и $\alpha, \alpha$ '-замещенные фураны (ди-, трифурилметаны) $[10,16]$. Реакции по $\beta$-положениям кольца протекают как окислительная конденсация, сопровождающаяся образованием простых эфиров [11]. Процессы могут протекать с участием СН-групп $\beta$-дикарбонильных производных, образующихся на стадиях щелочного распада. Последнее, вероятно, обусловливает более интенсивное и полное протекание указанных процессов в условиях сухой термодеструкции продуктов щелочного распада мальтозы.

Ключевыми интермедиатами дегидратации глюкозы являются $\alpha$-дикарбонильные производные ( $\alpha$-диулозы) - продукты деградации углеводных циклов [6], внутримолекулярная циклизация которых приводит к прекурсорам $\beta$-пирановых и $\alpha, \alpha^{\prime}, \beta$ - замещенных фурановых структур (фуранонов) [15], способных к дальнейшей внутри- и межмолекулярной дегидратации и конденсации с образованием высокомолекулярных продуктов. В общем виде структурные фрагменты образующихся карамелей можно представить следующим образом:

Таким образом, нерегулярная структура конденсированных продуктов термодеструкции включает различные фрагменты, идентификация которых возможна на основании анализа колебательных спектров. Кроме того, в последние годы развиваются хемометрические подходы, основанные на оценке структурно-группового состава гетерофункциональных продуктов по данным ИК Фурье-спектроскопии [17].

Ранее была показана возможность оценки структурных показателей карамелей в рамках двухпараметрической А-С-модели [19], значение параметров предлагается оценивать исходя из интенсивностей (Iv) соответствующих спектральных полос поглощения:

А-фактор характеризует алифатическую структурную составляющую $\left(2920 ; 2855 \mathrm{~cm}^{-1}-v_{\mathrm{C}-\mathrm{H}}\right)$, С-фактор - непредельную функциональную часть структуры карамелей. На основании полученных результатов построена зависимость (рис. 3, A), позволяющая наглядно сопоставить структурные особенности полученных продуктов.

Анализ данных рис. 3, А показывает преобладание ненасыщенных функционализированных фрагментов в структуре продуктов сухой термодеструкции мальтозы, что, вероятно, связано с меньшим количеством путей реакций в сравнении с деструкцией продуктов щелочного распада.

Сопоставляя полученные результаты с данными T. Schultz, J. Yarbrough, полученными ими при оценке токсичности $\alpha, \beta$-непредельных карбонильных соединений, можно прийти к выводу о взаимосвязи характера структурных элементов продуктов термодеструкции с их биоактивностью (см. рис. 3, В). Как следует из сопоставления данных, показатель токсичности $\mathrm{IGC}_{50}$ (концентрация 50 \%-го ингибирования роста тест-бактерий, мМ) модельных структур в реакции с SH-группами белков возрастает с увеличением непредельной функционализации и снижением предельной алифатической составляющей структуры. Слаботрансформированные продукты щелочного распада (см. рис. 3, А, точка 1) структурно соответствуют малотоксичным предельно-алифатическим структурам (см. рис. 3, В, точки I и II), тогда как продукты жесткой термодеструкции мальтозы (см. рис. 3, А, точка 3) функциально аналогичны более токсичным глубокотрансформированным непредельным оксопроизводным (см. рис. 3, В, точки V и VI). Подобный подход особенно перспективен для оценки структурных параметров продуктов трансформации углеводов, являющихся нелетучими высокомолекулярными веще- 
ствами, при этом их гетерофункциональность предполагает возможность построения более тонких корреляций на основании анализа соотношения интенсивностей выбранных полос поглощения в ИК-спектрах.

Таким образом, по результатам исследования можно сделать следующие выводы.

1. Методами ИК Фурье-спектроскопии и масс-спектрометрии показано, что в процессах щелочного распада и сухой карамелизации D-мальтозы формируются окрашенные структуры, содержащие предельные и непредельные алифатические фрагменты, конденсированные с гетероциклами различной степени замещенности.

2. Установлено, что сухая термодеструкция, требующая жесткого температурного режима, приводит к более глубоким структурным изменениям в процессе воздействия в сравнении с реакциями карамелизации в растворах. Показано преобладание непредельной и гетероциклической составляющей структуры продуктов сухой термодеструкции исходного углевода.

3. В рамках двухпараметрической модели показана возможность полуколичественной оценки структурных составляющих продуктов деструкции мальтозы в различных условиях: слаботрансформированные продукты щелочного распада структурно соответствуют малотоксичным предельно-алифатическим структурам, тогда как продукты жесткой термодеструкции мальтозы функциально аналогичны более токсичным глубокотрансформированным непредельным оксопроизводным.

4. Результаты исследований имеют практическое значение при исследовании структуры и прогнозировании свойств и характера биоактивности продуктов переработки углеводного сырья в пищевой промышленности.

\section{БИБЛИОГРАФИЧЕСКИЙ СПИСОК}

1. Urashima T., Suyama K, Adachi S. The formation of 1,6-anhydro-3,4-O-[5-(hydroxymethyl)2-furfurylidene]- $\beta$-D-galactopyranose from lactose during pyrolysis // Carbohydr. Res. - 1985. - Vol. 135. - P. 324-329.

2. Structural characterization and pyrolysis behavior of humin by-products from acid-catalyzed conversion of C6 and C5 carbohydrates / S. Wang, H. Lin, Y. Zhao [et al.] // J. Anal. Appl. Pyrol. 2016. - Vol. 118. - P. 259-266. - DOI: 10.1016/j.jaap.2016.02.009.

3. Zhao Z., Miao Y., Yang Z. Effect of sulfuric acid on the curing behavior and bonding performance of tannin-sucrose adhesive // Polymers. - 2018. - Vol. 10. - P. 651. - DOI: 10.3390/polym10060651.

4. Perez Locas C., Yaylayan $V$. Origin and mechanistic pathways of formation of parent furan - a food toxicant // J. Agric. Food Chem. - 2004. - Vol. 52. - P. 6830-6836.

5. Peterson $L$. Reactive metabolites in the biotransformation of molecules containing furan rings // Chem. Res. Toxicol. - 2013. - Vol. 26. - P. 5-26. - DOI: 10.1021/tx3003824.

6. Van der Kaaden A., Boon J., Haverkamp J. The analytical pyrolysis of carbohydrates. 2. Differentiation of homopolyhexoses according to their linkage type, by pyrolysis-mass spectrometry and pyrolysis-gas chromatography/mass spectrometry // Biomed. Mass Spectrom. - 1984. - Vol. 11. - P. 486-492.

7. Черепанов И.С. К вопросу о структуре продуктов карамелизации D-мальтозы в водно-этанольных средах // Вестник Южно-Уральского государственного университета. Серия: Пищевые и биотехнологии. - 2018. - № 2. - С. 14-19.

8. Kroh L. Caramelisation in food and beverages // Food Chem. - 1994. - Vol. 51. - P. 373-379. DOI: 10.1016/0308-8146(94)90188-0.

9. Biomass pyrolysis kinetics: a review of molecular-scale modeling contribution / J. Murillo, J. Biernacki, S. Northrup [et al.] // Braz. J. Chem. Eng. -2018. - Vol. 34. - P. 1-18. - DOI: 10.1590/01046632.20170341 s20160086. 
10. Patil S., Heltzel J., Lund C. Comparison of structural features of humins formed catalytically from glucose, fructose and 5-hydroxymethylfurfuraldehyde // Energy Fuels. - 2012. - Vol. 26. - P. 5281-5293. - DOI: 10.1021/ef3007454.

11. Shindo A., Izumino $K$. Structural variations during pyrolysis of furfuryl alcohol and furfurylfyrfuryl alcohol resins // Carbon. - 1994. - Vol. 32. - P. 1233-1243.

12. Edvards E., Oishi S., Botelho E. Analysis of chemical polymerization between functionalized MWCNT and poly (furfuryl alcohol) composite // Polimeros. - 2018. - Vol. 28. - P. 15-22. - DOI: 10.1590/0104-1428.07016.

13. Alakhras F., Holze R. In situ UV-vis- and FTIR-spectroscopy of electrochemically synthesized furan-thiophene copolymers // Synth. Met. - 2007. - Vol. 157. - P. 109-119.

14. Spectroscopic characterization of $\alpha$ - and $\gamma$-pyrones and their substituted 4-hydroxy and 4-methoxy derivatives: an integrated infrared, photophysical and theoretical study / J. Seikas de Melo, G. Quenteiro, J. Pina [et al.] // J. Mol. Struct. - 2001. - Vol. 565-566. - P. 59-67.

15. Ledl F., Hiebl J., Severin T. Bulding farbiger $\beta$-pyranone aus pentoses und hexoses // Z. Lebensm. Unters. Forsch. - 1983. - Vol. 177. - P. 353-355.

16. Curing mechanism of furan resin modified with different agents and their thermal strength / H. Renhe, G. Hongmei, T. Yaoji [et al.] // Research \& Development. - 2011.- Vol. 8. - P. 162-165.

17. Formation, molecular structure, and morphology of humins in biomass conversion: influence of feedstock and processing conditions / I. van Zandvoort, Y. Wang, C. Rasrendra [et al.] // ChemSusChem. - 2013. - Vol. 6. - P. 1745-1758. - DOI: 10.1002/cssc.201300332.

18. Activation of cellulose via cooperative hydroxyl-catalyzed transglycosilation of glycosidic bonds / V. Maliekkal, S. Maduskar, D. Saxon [et al.] // ACS Catal. - 2019. - Vol. 9. - P. 1943-1955. DOI: $10.1021 /$ acscatal.8b04289.

19. Черепанов И.С., Красноперова О.С. О возможности количественной оценки структуры продуктов карамелизации сахаров методом ИК Фурье- спектроскопии // Технология и товароведение инновационных пищевых продуктов. - 2019. - № 3. - С. 3-8.

20. Schultz T., Yarbrough J. Trends in structure-toxicity relationship for carbonyl-containing $\alpha$, $\beta$-unsaturated compounds // SAR QSAR Environ. Res. - 2004. - Vol. 15. - P. 139-146.

\section{REFERENCES}

1. Urashima T., Suyama K, Adachi S. The formation of 1,6-anhydro-3,4-O-[5-(hydroxymethyl)2 -furfurylidene]- $\beta$-D-galactopyranose from lactose during pyrolysis, Carbohydr. Res, 1985, vol. 135, pp. 324-329.

2. Wang S., Lin H., Zhao Y., Zhang J.-F. Structural characterization and pyrolysis behavior of humin by-products from acid-catalyzed conversion of C6 and C5 carbohydrates, J. Anal. Appl. Pyrol, 2016, vol. 118, pp. 259-266, DOI: 10.1016/j.jaap.2016.02.009.

3. Zhao Z., Miao Y., Yang Z. Effect of sulfuric acid on the curing behavior and bonding performance of tannin-sucrose adhesive, Polymers, 2018, vol. 10, pp. 651, DOI: 10.3390/polym10060651.

4. Perez Locas C., Yaylayan V. Origin and mechanistic pathways of formation of parent furan - a food toxicant, J. Agric. Food Chem, 2004, vol. 52, pp. 6830-6836.

5. Peterson L. Reactive metabolites in the biotransformation of molecules containing furan rings, Chem. Res. Toxicol, 2013, vol. 26, pp. 5-26, DOI: 10.1021/tx3003824.

6. Van der Kaaden A., Boon J., Haverkamp J. The analytical pyrolysis of carbohydrates. 2. Differentiation of homopolyhexoses according to their linkage type, by pyrolysis-mass spectrometry and pyrolysis-gas chromatography/mass spectrometry, Biomed. Mass Spectrom, 1984, vol. 11, pp.486-492. 
7. Cherepanov I.S. Vestnik Yujno-Ural'skogo gosudarstvennogo universiteta, 2018, No. 2, pp. 14-19. (In Russ.)

8. Kroh L. Caramelisation in food and beverages, Food Chem, 1994, vol. 51, pp. 373-379, DOI: 10.1016/0308-8146(94)90188-0.

9. Murillo J., Biernacki J., Northrup S., Mohammad A. Biomass pyrolysis kinetics: a review of molecular-scale modeling contribution, Braz. J. Chem. Eng, 2018, vol. 34, pp. 1-18, DOI: 10.1590/0104-6632.20170341s20160086.

10. Patil S., Heltzel J., Lund C. Comparison of structural features of humins formed catalytically from glucose, fructose and 5-hydroxymethylfurfuraldehyde, Energy Fuels, 2012, vol. 26, pp. 52815293, DOI: 10.1021/ef3007454.

11. Shindo A., Izumino K. Structural variations during pyrolysis of furfuryl alcohol and furfurylfyrfuryl alcohol resins, Carbon, 1994, vol. 32, pp. 1233-1243.

12. Edvards E., Oishi S., Botelho E. Analysis of chemical polymerization between functionalized MWCNT and poly (furfuryl alcohol) composite, Polimeros, 2018, vol. 28, pp. 15-22, DOI: 10.1590/0104-1428.07016.

13. Alakhras F., Holze R. In situ UV-vis- and FTIR-spectroscopy of electrochemically synthesized furan-thiophene copolymers, Synth. Met, 2007, vol. 157, pp. 109-119.

14. Seixas J. de Pina, Melo J., Quinteiro G., Breda J., Fausto S. Spectroscopic characterization of $\alpha$ - and $\gamma$-pyrones and their substituted 4-hydroxy and 4-methoxy derivatives: an integrated infrared, photophysical and theoretical study, J. Mol. Struct, 2001, vol. 565-566, pp. 59-67.

15. Ledl F., Hiebl J., Severin T. Bulding farbiger $\beta$-pyranone aus pentoses und hexoses, Z. Lebensm. Unters. Forsch, 1983, vol. 177, pp. 353-355.

16. Renhe H., Hongmei G., Yaoji T., Qingyun L. Curing mechanism of furan resin modified with different agents and their thermal strength, Research \& Development, 2011, vol. 8, pp. 162-165.

17. Zandvoort I., Wang Y., Rasrendra C., Ernst R.H. van Eck, Pieter C.A. Bruijnincx, Heeres H.J., Weckhuysen Bert M. Formation, molecular structure, and morphology of humins in biomass conversion: influence of feedstock and processing conditions, ChemSusChem, 201, vol. 6, pp. 17451758, DOI: $10.1002 / \mathrm{cssc} .201300332$.

18. Maliekkal V., Maduskar S., Saxon D., Nasiri M., Reineke T.M., Neurock M., Dauenhauer P. Activation of cellulose via cooperative hydroxyl-catalyzed transglycosilation of glycosidic bonds, ACS Catal, 2019, vol. 9, pp. 1943-1955, DOI: 10.1021/acscatal.8b04289.

19. Cherepanov I.S., Krasnoperova O.S. Tekhnologiya I tovarovedeniye innvaatsionnykh pitschevykh produktov, 2019, No. 3, pp. 3-8. (In Russ.)

20. Schultz T., Yarbrough J. Trends in structure-toxicity relationship for carbonyl-containing $\alpha$, $\beta$-unsaturated compounds, SAR QSAR Environ. Res, 2004, vol. 15, pp. 139-146. 